\title{
The Real Time Implementation of a Chaotic System's Synchronization for Secure Communication
}

\author{
Hasan GULER, Vedat CELIK, Turgay KAYA, Yavuz EROL
}

\begin{abstract}
The chaotic systems are preferred in secure communication systems as well as used in many different implementation areas. Real time chaos synchronization is also required for secure communication systems. In this study, the Master-Slave synchronization of chaotic Chen system was performed on a real time implementation for the secure communication systems. Active control method was used in the design of controller required for the realization of synchronization. The successful realization of the Master-Slave synchronization of the Chen system by using the controller designed on MATLAB/Simulink was shown by the obtained simulation results. Moreover, by using analogue outputs of NI-DAQ card, the Master-Slave synchronization of the Chen system was obtained and secure communication was achieved on a real time basis under LabVIEW environment.
\end{abstract}

Keywords: active control; chaotic Chen system; Master-Slave synchronization; secure communication; LabVIEW

\section{INTRODUCTION}

Chaos is defined as periodic behaviours which exhibit sensitive dependence on initial conditions in a deterministic system [1]. It is known that this behaviour form arises in continuous [2-4], discrete [5-6] and timedelayed [7-8] systems. This behaviour observed in nonlinear systems is used in different areas such as cryptology [9], driver circuits [10], increasing effectiveness of heuristic optimization methods [11], random number generators [12-13] and secure communication systems [14-16].

The trajectories of the same system operating at different initial conditions within chaotic systems will move away in time due to sensitive dependence on initial conditions. In 1990, after Pecora and Carroll showed that a chaotic system can be synchronized [17], various methods started to be used in order to converge these two different trajectories [18-22]. By using these methods, when two chaotic systems in the form of Master-Slave are defined, the Slave system will start to follow-up the Master system at any time required. This operation is called chaotic synchronization [17]. Thus the system consisting of receiver and transmitter will be able to be used in secure communication systems. Two chaotic systems as Master (Transmitter) and Slave (Receiver) constitute the two main parts of secure communication. In order to ensure the communication performed between these two main parts, the achievement of synchronization is required. Thus, it is needed to implement the synchronization of chaotic systems and to apply it on real time. Recently, studies both on the real time implementations of chaotic systems [23-24] and on the real time synchronization of chaotic systems [25-26] have started to be performed.

For real time implementations, LabVIEW program is preferred in many areas such as biomedical, control, communication etc. [27-31]. In this study, the reason of preferring the LabVIEW program is its graphic based structure and the ease provided to user in constituting interface. Moreover, the real time implementations are able to be performed easily by the hardware such as DAQ card and cRIO. Recently, both the simulation [32] and real time implementations [33] of chaotic systems have been achieved on LabVIEW environment.

In literature, the studies performed regarding chaotic systems are generally simulation based. But the studies performed for the real time implementations of such systems are limited. The real time implementations of chaotic systems had started to increase depending on technological developments. In this study, for the real time implementations of secure communication systems, the Master-Slave synchronization of the chaotic Chen system indicated in [4] was implemented by the active control method provided in [18-19], and their simulations and real time implementations were presented on MATLAB/Simulink and LabVIEW environment, respectively. Along with this introduction, chaotic Chen system was presented in Section 2, and suitable controller enabling chaotic synchronization by active control method was determined. In Section 3, by the designed controlled, the simulations and Master-Slave synchronization were performed on MATLAB/Simulink and on LabVIEW environment respectively, and the results of the real time Master-Slave synchronization of the Chen system were obtained and the secure communication was implemented by using a sample data signal. Finally, the results were discussed in Section 4.

\section{MATERIALS AND METHODS \\ 2.1 Chaotic Chen System}

The Chen system presented in [4] is a third degree non-linear autonomous system, and the state-space representation is defined as Eq. (1).

$$
\begin{aligned}
& \dot{x}=a(y-x) \\
& \dot{y}=(c-a) x-x z+c y, \\
& \dot{z}=x y-b z
\end{aligned}
$$

where, $a, b$ and $c$ are positive system parameters, and $x, y$ and $z$ are state variables. The system defined in Eq. (1) is able to present chaotic behaviour depending on the system parameters [4]. State-space diagram on the $x-y-z$ plain of the Chen system is given in Fig. 1. 
As it is seen in Fig. 1, this system shows chaotic behaviour for $a=35, b=3$ and $c=28$. These parameters were used in simulations and real time implementations obtained in the following sections.

$$
a=35, b=3, c=28
$$

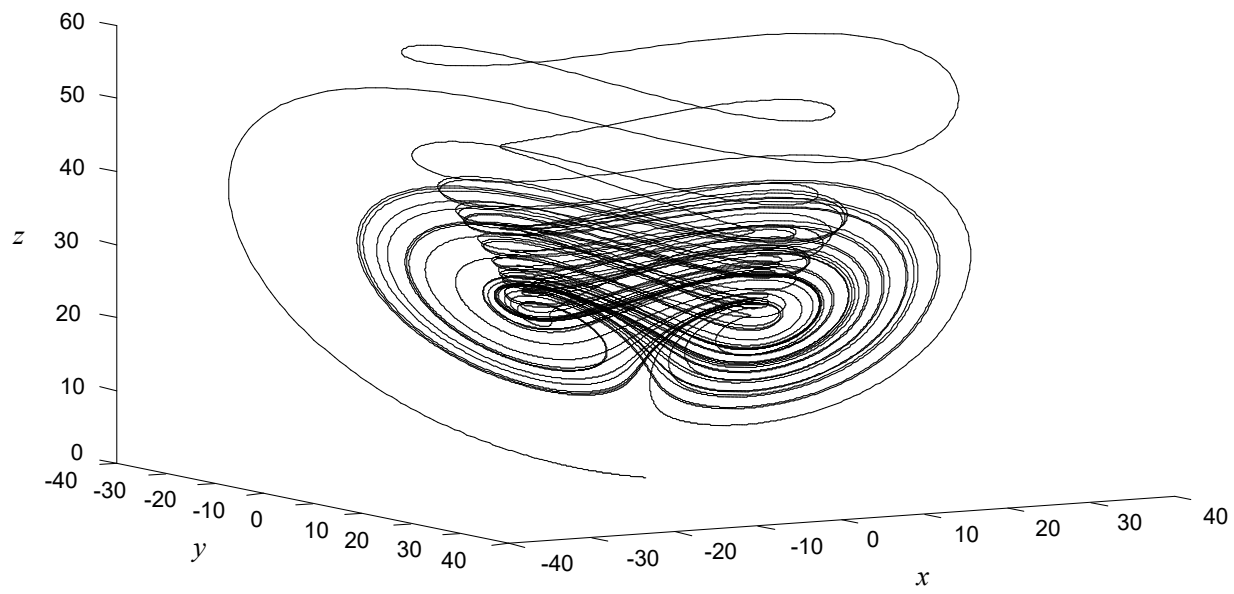

Figure 1 The state-space diagram of the chaotic Master Chen System

\subsection{The Master-Slave Synchronization of the Chen System via Active Control}

In order to fulfil the chaotic synchronization of the Chen system by the active control method provided in [18-19], it will be required to obtain the error dynamic of its outputs at different initial conditions. In Eq. (2), the model of Master Chen system, and in (3), the models of the system consisting of Slave Chen system and control function are given.

$$
\begin{aligned}
& \dot{x}_{m}=a\left(y_{m}-x_{m}\right) \\
& \dot{y}_{m}=(c-a) x_{m}-x_{m} z_{m}+c y_{m} \\
& \dot{z}_{m}=x_{m} y_{m}-b z_{m} \\
& \dot{x}_{s}=a\left(y_{s}-x_{s}\right)+\mu_{1}(t) \\
& \dot{y}_{s}=(c-a) x_{s}-x_{s} z_{s}+c y_{s}+\mu_{2}(t) \\
& \dot{z}_{s}=x_{s} y_{s}-b z_{s}++\mu_{3}(t)
\end{aligned}
$$

where, $\mu_{1}(t), \mu_{2}(t)$ and $\mu_{3}(t)$ are the control functions. The phrase defining the errors of the two systems given in Eqs. (2) and (3) are expressed in Eq. (4) as $e_{1}=x_{s}-x_{m}, e_{2}$ $=y_{s}-y_{m}$ and $e_{3}=z_{s}-z_{m}$.

$$
\begin{aligned}
& \dot{e}_{1}=a\left(e_{2}-e_{1}\right)+\mu_{1}(t) \\
& \dot{e}_{2}=(c-a) e_{1}+c e_{2}-x_{s} z_{s}+x_{m} z_{m}+\mu_{2}(t) \\
& \dot{e}_{3}=x_{s} y_{s}-x_{m} y_{m}-b e_{3}+\mu_{3}(t)
\end{aligned}
$$

If the control signals are selected to eliminate nonlinear components as the active control functions being $V_{1}(t)=-k_{1} e_{1}, V_{2}(t)=-k_{2} e_{2}$ and $V_{3}(t)=-k_{3} e_{3}$.

$$
\begin{aligned}
& \mu_{1}(t)=V_{1}(t) \\
& \mu_{2}(t)=x_{s} z_{s}-x_{m} z_{m}+V_{2}(t) \\
& \mu_{3}(t)=x_{m} y_{m}-x_{s} y_{s}+V_{3}(t)
\end{aligned}
$$

the error dynamic will turn to a linear structure given in Eq. (6).

$$
\begin{aligned}
& \dot{e}_{1}=a\left(e_{2}-e_{1}\right)+V_{1}(t) \\
& \dot{e}_{2}=(c-a) e_{1}+c e_{2}+V_{2}(t) \\
& \dot{e}_{3}=-b e_{3}+V_{3}(t)
\end{aligned}
$$

In the Matrix form,

$$
\left[\begin{array}{l}
\dot{e}_{1} \\
\dot{e}_{2} \\
\dot{e}_{3}
\end{array}\right]=\left[\begin{array}{ccc}
-\left(a+k_{1}\right) & a & 0 \\
c-a & c-k_{2} & 0 \\
0 & 0 & -\left(b+k_{3}\right)
\end{array}\right]\left[\begin{array}{l}
e_{1} \\
e_{2} \\
e_{3}
\end{array}\right]
$$

by using the coefficient matrix of Eq. (7), the characteristic equation can be obtained as follows.

$$
\begin{aligned}
& \left(\lambda+\left(b+k_{3}\right)\right)\left(\lambda^{2}+\left(a-c+k_{1}+k_{2}\right) \lambda+a k_{2}-\right. \\
& \left.-2 a c+k_{1} k_{2}-c k_{1}+a^{2}\right)=0
\end{aligned}
$$

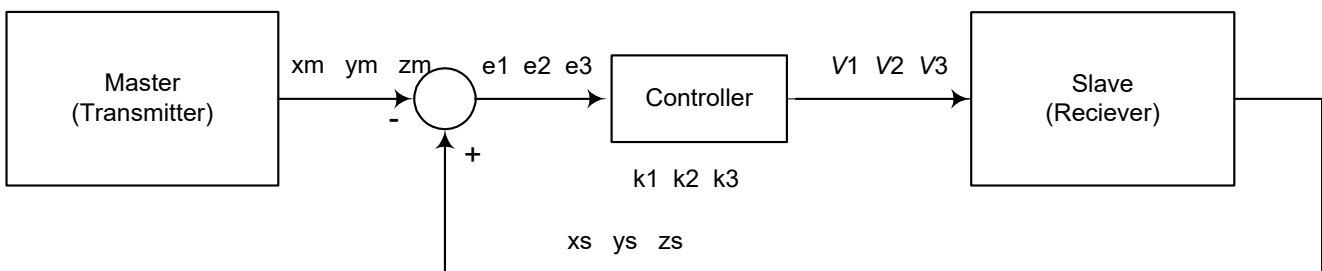

Figure 2 The block diagram of the master-slave synchronization

Depending on the parameters of the Chen system, there are many active controller gain values which will enable all the eigenvalues of Eq. (8) to be on the left-half s-plane. If $k_{1}=2, k_{2}=30$ and $k_{3}=10$ are selected for the 
chaotic parameters of the system $a=35, b=3$ and $c=28$, the eigenvalues of the error dynamic are obtained as $\lambda_{1}=$ $-27.32, \lambda_{2}=-11.67$ and $\lambda_{3}=-13$. Thus, the gain values of the controller achieved synchronization can be taken as selected values due to the stable error dynamic. In Fig. 2, by using active control, the block diagram of the MasterSlave synchronization of identical two chaotic Chen systems is shown.

\section{IMPLEMENTATION OF THE MASTER-SLAVE SYNCHRONIZATION OF CHEN SYSTEM BY USING LabVIEW}

In this section, the simulation of the chaotic Chen system on MATLAB/Simulink and real time synchronization of the chaotic Chen system under LabVIEW environment were implemented by using the obtained controller's parameters in order to ensure Master-Slave synchronization. Moreover, an example for secure communication was performed. For this, while taking the initial conditions of the Master system as $\left(x_{m}(0), y_{m}(0), z_{m}(0)\right)=(0.1,0,0)$, the initial conditions of the Slave system were selected close to each other as $\left(x_{s}(0), y_{s}(0), z_{s}(0)\right)=(0.1001,0,0)$. If the difference in between the initial conditions of the Master and the Slave system is looked at closely, it is $1 / 1000$.
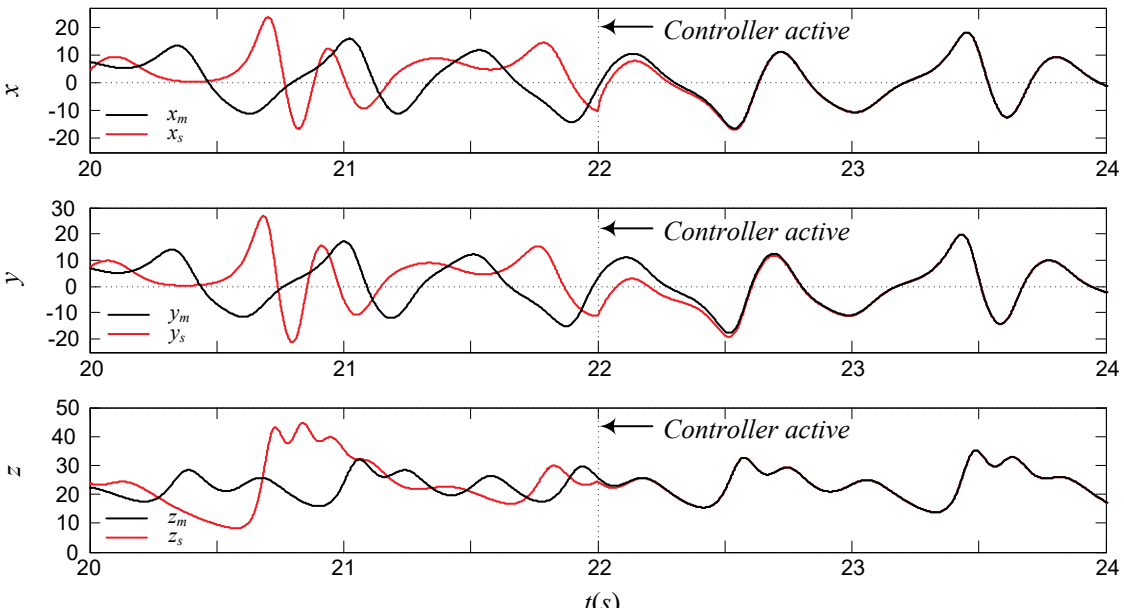

Figure 3 The time responses: the state variables of the Master system and the Slave systems
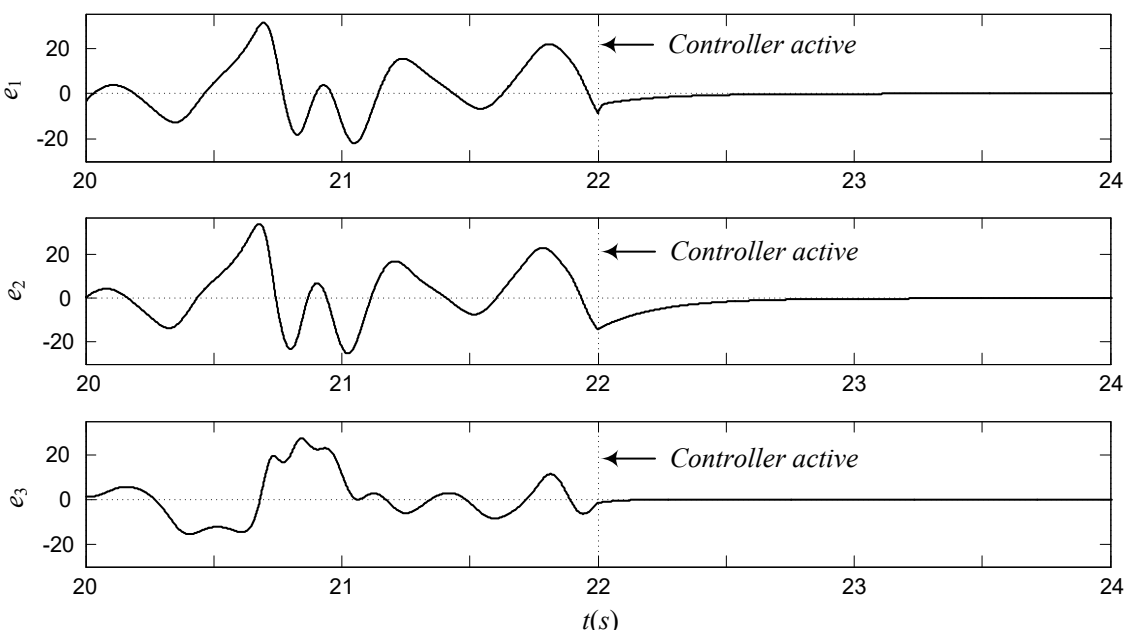

Figure 4 The time responses: the error of the state variables between Master and Slave systems

\subsection{The Simulation Results of the Master-Slave Synchronization on MATLAB/Simulink}

First of all, the Master and Slave parts of chaotic Chen system were constituted on MATLAB/Simulink and the simulation was achieved for the synchronization of the system. In the simulations, the controller was activated in $22^{\text {nd }}$ second and the synchronization was tried to be performed. The changes of the Master and the Slave systems for each state variable and the error in between them are shown in Fig. 3 and 4 respectively.

As it is seen, the Slave system follows the Master system in a short time due to the controller which is activated in $22^{\text {nd }}$ second. Since all the eigenvalues of the error dynamic are on the left-half s-plane and on $\sigma$ axis for these controller gains, the error goes to zero exponentially. 


\subsection{The Real Time Results of the Master-Slave Synchronization on LabVIEW}

NI-6009 DAQ card was used for real time implementation of the Chen systems. NI-6009 DAQ card having 8 analogue inputs (14-bit, $48 \mathrm{kS} / \mathrm{s}$ ), 2 analogue outputs (12-bit, $150 \mathrm{~S} / \mathrm{s}$ ) and 12 digital I/O, and 32-bit counter was used in the real time implementation of the chaotic Chen system. In this implementation, since the analogue output of DAQ card is able to provide a voltage of $0-5 \mathrm{~V}$, the z-state variable output was weakened by 20 times and a DC component signal of $0.1 \mathrm{~V}$ was added to this signal in order to prevent the signal to go to negative.

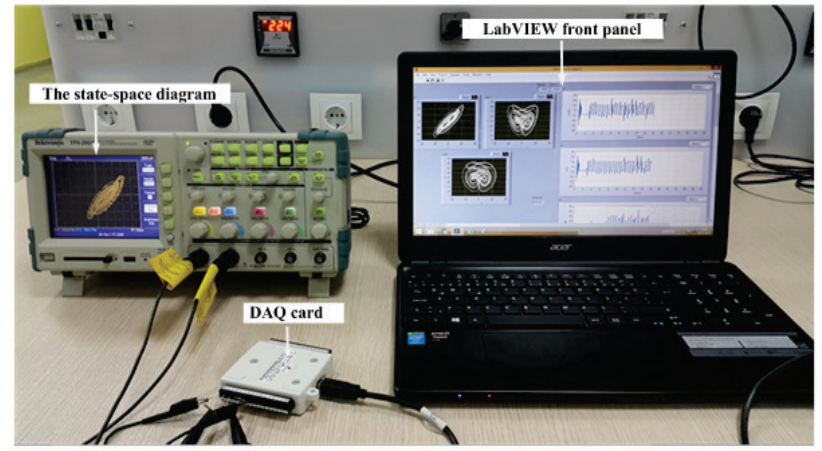

Figure 5 The general appearance of the experimental system The general appearance of the designed experimental system is shown in Fig. 5.

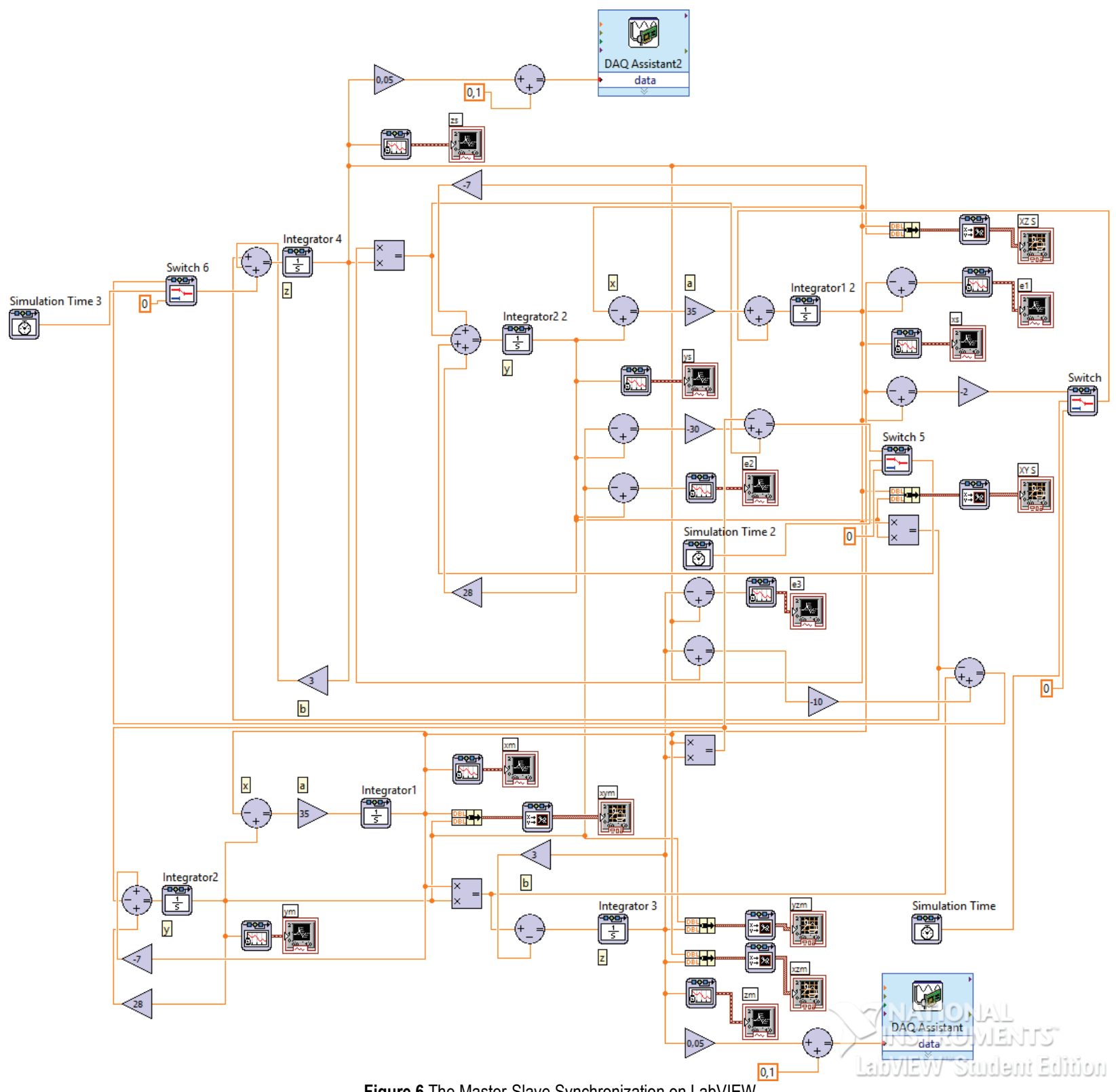

Figure 6 The Master-Slave Synchronization on LabVIEW

The Master and Slave parts of chaotic Chen system were constituted on LabVIEW environment. The program of the implemented system was given in Fig. 6. For the synchronization of the system, the parameters of the designed controller in the previous section were integrated to this system. In Fig. 7, the obtained state- space diagrams of the chaotic master system in real time were given for the $x-y, x-z$ and $y-z$ planes.

The oscilloscope view obtained before and after the synchronization of Master and Slave system of the $z$-state variable was given in Fig. 8. In this figure, the real time master and slave systems were represented as yellow and 
blue lines, respectively. Also, in the same figure, the change of error between these two systems of the $z$-state variable obtained on the oscilloscope was shown as red line.

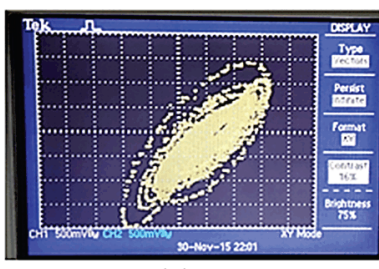

(a)

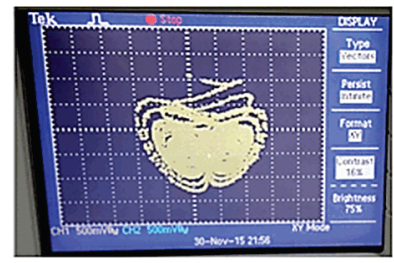

(b)

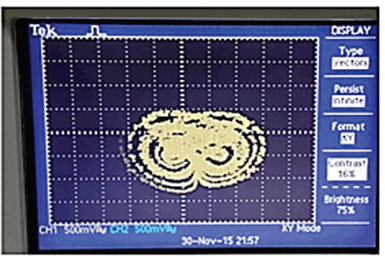

(c)

Figure 7 The state-space diagrams of the chaotic Master Chen System for a) $x$ $y$, b) $x-z$ and c) $y-z$ planes

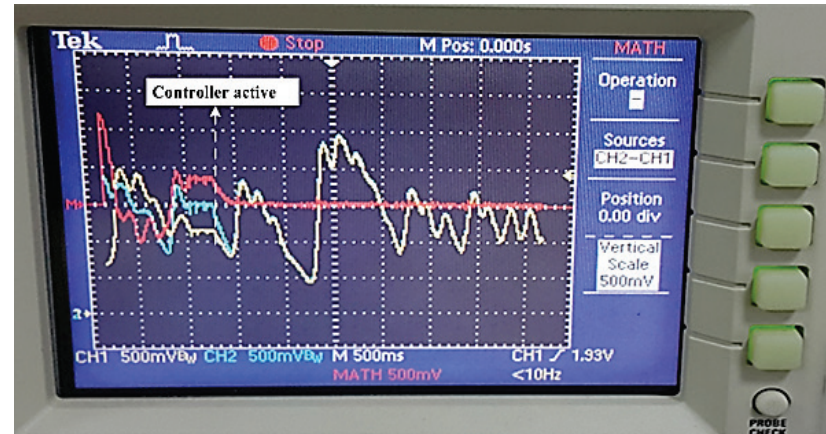

Figure 8 Responses of the Master system (yellow), the Slave system (blue) and the error between these systems (red) of the $z$-state variable in real-time.

As it is seen, the output of Slave system of the $z$-state variable has started to follow-up the Master system by the activation of controller. In addition, the change of error between these two systems exponentially goes to zero.

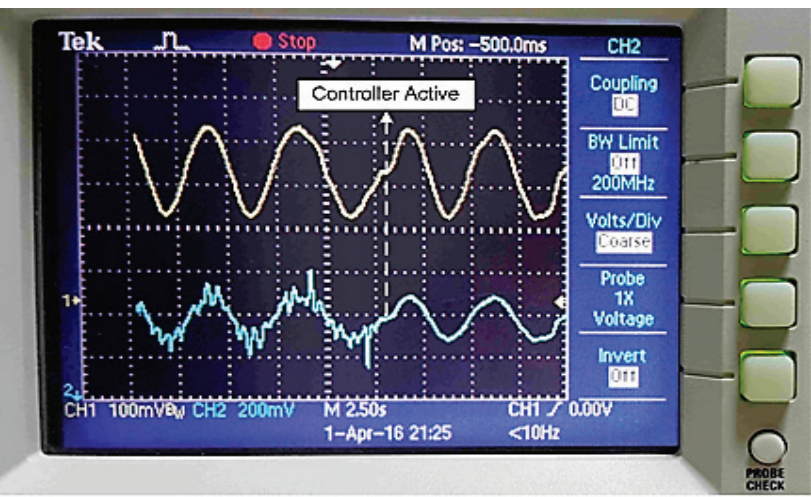

Figure 9 The sample data signal (yellow) and the demodulated signal (blue).

In addition, the secure communication example was performed by using Master-Slave synchronization system. A data signal was chosen for sinusoidal signal with 0.2 $\mathrm{Hz}$ frequency and $0.1 \mathrm{~V}$ amplitude. The sample data signal for the secure communication system was modulated with the signal obtained from Master system. Then, the transmitted signal was demodulated with Slave system. The variations of sample data signal and the demodulated signal were given in Fig. 9 and the modulated and the demodulated signal in Fig 10.

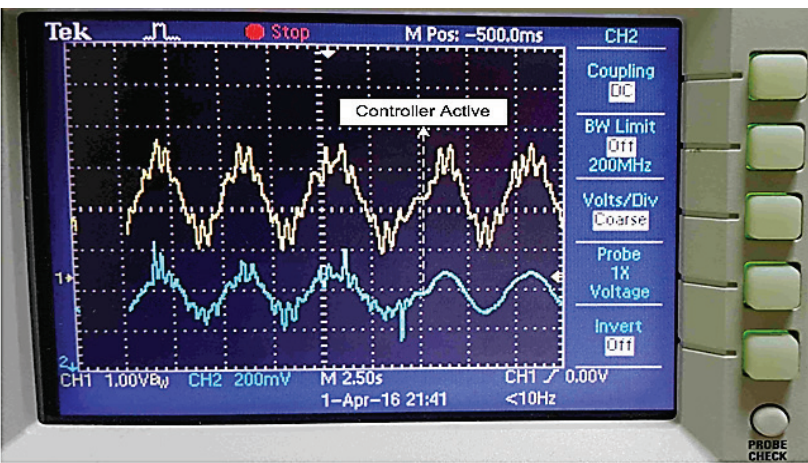

Figure 10 The modulated signal (yellow) and the demodulated signal (blue)

As can be seen from these figures, although the modulated signal was demodulated signal with Slave system signal, the data signal was not obtained until the controller was active.

\section{DISCUSSION AND CONCLUSIONS}

Generally, simulation studies have been performed regarding chaotic systems. However the studies performed for the real time implementations of such systems are few. By the software and hardware facilitating the real time implementations such as LabVIEW, the possibility of implementing chaotic systems has increased. In this study, a controller was designed to use the Master-Slave synchronization of the chaotic Chen system with the active control method in secure communication systems. By using this controller, the Master-Slave synchronization of the Chen system on MATLAB/Simulink environment was simulated, and it was observed that for all three state variables, the Slave systems followed the Master system in a short time while after the activation of the controller, and that the error in between two systems has gone to zero exponentially. In addition, using the designed controller on LabVIEW, the synchronization of the Chen system was obtained on a real time by DAQ card. At the output of DAQ card, the error signal between the Master and Slave systems was obtained, and the implementation of the real time synchronization was observed. Moreover, a secure communication example was achieved by using the Master-Slave system.

Consequently, according to the obtained results, it is considered that the LabVIEW environment will provide significant ease in the performance of real-time implementation of various chaotic systems of such secure communication.

\section{REFERENCES}

[1] Strogatz, S. H. (1994). Nonlinear Dynamics and Chaos, Westview Press.

[2] Lorenz, E. N. (1963). Deterministic nonperiodic flow. $J$. Atmos. Sci., 20, 130-141. https://doi.org/10.1175/1520-0469(1963)020<0130:DNF>2.0.C0;2

[3] Huang, J., Li, C., \& He, X. (2013). Stabilization of a Memristor-based Chaotic System by Intermittent Control and Fuzzy Processing. Int. Journal of Control, Automation and Systems, 11(3), 643-647. 
https://doi.org/10.1007/s12555-012-9323-x

[4] Chen, G. \& Ueta, T. (1999). Yet another chaotic attractor. Int. J. Bifurc. Chaos, 9, 1465-1466. https://doi.org/10.1142/S0218127499001024

[5] Feigenbaum, M. J. (1978). Quantitative Universality for a Class of Non-Linear Transformations. J. Stat. Phys., 19, 2552. https://doi.org/10.1007/BF01020332

[6] Hénon, M. (1976). A two-dimensional mapping with a strange attractor. Communications in Mathematical Physics, 50(1), 69-77. https://doi.org/10.1007/BF01608556

[7] Mackey, M. C. \& Glass L. (1977). Oscillation and chaos in physiological control systems. Science, 197, 287-289. https://doi.org/10.1126/science.267326

[8] Uçar, A. (2002). A prototype model for chaos studies. Int. J. Eng. Sci., 40, 251-258. https://doi.org/10.1016/S0020-7225(01)00060-X

[9] Özkaynak, F. \& Özer, A. B. (2010). A method for designing strong S-Boxes based on chaotic Lorenz system. Physics Letters A, 374(36), 3733-3738. https://doi.org/10.1016/j.physleta.2010.07.019

[10] Asker, M. E., Kürüm, H., \& Özer, A. B. (2015). Reduction of EMI by Using Chaotic Sinusoidal PWM on Vector Controlled PMSM. International Journal of Scientific and Technological Research, 1(1), 83-93.

[11] Alatas, B., Akin, E., \& Özer, A. B. (2009). Chaos embedded particle swarm optimization algorithms. Chaos, Solitons \& Fractals, 40(4), 1715-1734.

[12] Tuncer, T., Avaroğlu, E., Türk, M., \& Özer, A. B. (2014). Implementation of non-periodic sampling true random number generator on FPGA. J. Microelectron. Electron. Compon. Mater., 44(4), 296-302. https://doi.org/10.1016/j.chaos.2007.09.063

[13] Avaroğlu, E., Tuncer, T., Özer, A. B., \& Türk, M. (2014). A new method for hybrid pseudo random number generator. J. Microelectron. Electron. Compon. Mater., 4(4), 303-311.

[14] Cuomo, K. M., Oppenheim, A. V., \& Strogatz, S. H. (1993). Synchronization of Lorenz-Based Chaotic Circuits with Applications to Communications. IEEE Trans. on CAS-II, 40(10), 626-633. https://doi.org/10.1109/82.246163

[15] Yang, T. \& Chua, L. O. (1996). Secure Communication via Chaotic Parameter Modulation. IEEE Trans. on CAS-I, 43(9), 817-819. https://doi.org/10.1109/81.536758

[16] Grzybowski, J. M. V., Rafikov, M., \& Balthazar, J. M. (2009). Synchronization of the unified chaotic system and application in secure communication. Commun. Nonlinear Sci. Numer. Simulat., 14, 2793-2806. https://doi.org/10.1016/j.cnsns.2008.09.028

[17] Pecora, L. M. \& Carrol T. L. (1990). Synchronization in chaotic systems. Phys. Rev. Lett., 64, 821-824. https://doi.org/10.1103/PhysRevLett.64.821

[18] Bai, E. W. \& Lonngren, K. E. (1997). Synchronization of two Lorenz systems using active control. Chaos Solitons and Fract., 8(1), 51-58. https://doi.org/10.1016/S0960-0779(96)00060-4

[19] Uçar, A., Lonngren, K. E., \& Bai, E.W. (2003). Synchronization of chaotic behavior in nonlinear Bloch equations. Physics Letters A, 314, 96-101. https://doi.org/10.1016/S0375-9601(03)00864-8

[20] Wang, C. \& Ge, S. (2001). Adaptive backstepping control of uncertain Lorenz system. Int. J. Bifurc. Chaos, 11, 11151119. https://doi.org/10.1142/S0218127401002560

[21] Hua, C. \& Guan, X. (2005). Synchronization of chaotic systems based on PI observer design. Phys. Lett. A, 334, 382-389. https://doi.org/10.1016/j.physleta.2004.11.050

[22] Noroozi, N., Roopaei, M., \& Karimaghaee, P. (2009). Adaptive control and synchronization in a class of partially unknown chaotic systems. Chaos, 19, 023121. https://doi.org/10.1063/1.3155069
[23] Sadoudi, S., Azzaz, M. S., Djeddou, M., \& Benssalah, M. (2009). An FPGA Real-time Implementation of the Chen's Chaotic System for Securing Chaotic Communications. International Journal of Nonlinear Science, 7(4), 467-474.

[24] Koyuncu, İ., Özcerit, A. T., \& Pehlivan, İ. (2014). Implementation of FPGA-based real time novel chaotic oscillator. Nonlinear Dyn., 77, 49-59. https://doi.org/10.1007/s11071-014-1272-x

[25] Rodriguez-Bollain, A., Mata-Machuca, J. L., \& MartinezGuerra, R. (2010). Synchronization of Chaotic Systems: A Real-Time Application to Colpitts Oscillator. CCE $2010 /$ Mexico, 60-65,

[26] Azzaz, M. S., Tanougast, C., Sadoudi, S., Bourıdane, A., \& Dandache, A. (2010). An FPGA Implementation of a FeedBack Chaotic Synchronization for Secure Communications. CSNDSP 2010 / Newcastle, 239-243.

[27] Guler, H., Turkoglu, I., \& Ata, F. (2014). Designing Intelligent Mechanical Ventilator and User Interface Using LabVIEW $^{\circledR}$. Arabian Journal for Science and Engineering, 39(6), 4805-4813. https://doi.org/10.1007/s13369-014-1090-y

[28] Guler, H. \& Ata, F. (2014). The Comparison of Manual and LabVIEW-Based Fuzzy Control on Mechanical Ventilation. Proceedings of the Institution of Mechanical Engineers Part H-Journal of Engineering in Medicine, 228(9), 916-925. https://doi.org/10.1177/0954411914550513

[29] Guler, H. \& Ata, F. (2014). Design of a Fuzzy-LabVIEWBased Mechanical Ventilator. Computer Systems Science and Engineering, 29(3), 219-229.

[30] Poorna-chandra B. R., Geevarghese, K. P., \& Gangadharan, K. V. (2014). Design and Implementation of Remote Mechatronics Laboratory for e-Learning Using LabVIEW and Smartphone and Cross-platform Communication Toolkit (SCCT). Procedia Technology, 14, 108-115. https://doi.org/10.1016/j.protcy.2014.08.015

[31] Yu Y., Zhang, Y., Yuan, X., \& Hou, Q. (2014). A LabVIEW-based real-time measurement system for polarization detection and calibration. Optik, 125, 22562260. https://doi.org/10.1016/j.ijleo.2013.10.079

[32] Gupta, J., Kosta, S. P., \& Mor, P. (2011). Simulation of non-autonomous chaotic circuit on LabVIEW using nonlinear electrolytic device. Int. J. of Electronics Engineering, 3(2), 215-220.

[33] Elkholy, M. M., EL Hennawy, H. M., \& Elkouny, Abdellatif. (2015). Simulation and Digital Implementation of Eight Dimensional Hyper Chaotic System for Secured Chaotic Communication. Int. J. of Eng. Research \& Technology (IJERT), 4(3), 482-486.

\section{Contact information:}

Hasan GULER, PhD

Firat University, Engineering Faculty, Electrical-Electronics Engineering Department, 23119, Elazig/Turkey

hasanguler@firat.edu.tr

\section{Vedat CELIK, PhD}

Firat University, Engineering Faculty, Electrical-Electronics Engineering Department, 23119, Elazig/Turkey

celik@firat.edu.tr

Turgay KAYA, PhD

Firat University, Engineering Faculty, Electrical-Electronics Engineering Department, 23119, Elazig/Turkey

tkaya@firat.edu.tr

Yavuz EROL, PhD

Firat University, Engineering Faculty, Electrical-Electronics Engineering Department, 23119, Elazig/Turkey

yerol@firat.edu.tr 